\section{Transtornos mentais maternos graves e risco de malformação congênita do bebê: uma metanálise}

\author{
Severe mental illness in mothers and congenital \\ malformations in newborns: a meta-analysis
}

\author{
1 Instituto de Estudos em \\ Saúde Coletiva, Universidade \\ Federal do Rio de Janeiro, Rio \\ de Janeiro, Brasil. \\ Correspondência \\ P. K. Pereira \\ Instituto de Estudos em \\ Saúde Coletiva, Universidade \\ Federal do Rio de Janeiro. \\ Praça Jorge Machado \\ Moreira, Ilha do Fundão, \\ Cidade Universitária, Rio de \\ Janeiro, RJ 21944-970, Brasil. \\ priscilakrauss@ig.com.br
}

\section{Abstract}

The risk of congenital malformations appears to be higher in infants of mothers with mental disorders as compared to those of mothers with no history of psychiatric illness. This article presents a meta-analysis of studies on the association between maternal mental illness and congenital malformations. The review consisted of an article search in the MEDLINE, ISIWEB, Scopus, and SciELO databases, using the following key words: "mental disorders" $O R$ "mental health" $O R$ "psychotic disorders" $O R$ "schizophrenia" $A N D$ "congenital abnormalities" OR "birth defects". A total of 108 studies were identified, and five articles were selected according to the established criteria. These articles were included in a meta-analysis, involving a total of 4,194 children of mothers with mental illness and 249,548 children of mothers with no such disorders. Pooled relative risk showed a significant association between exposure to mental illness in mothers and risk of malformations in newborns $(R R=2.06$, 95\%CI: 1.46-2.67). The study highlights the relationship between maternal mental health during pregnancy and its effects on the infant's health.

Congenital Abnormalities; Mental Disorders; Schizophrenia; Meta-Analysis
Priscila Krauss Pereira 1

Lúcia Abelha Lima ${ }^{1}$

Mônica Maria Ferreira Magnanini 1

Leticia Fortes Legay 1

Giovanni Marcos Lovisi 1

\section{Introdução}

A associação entre transtornos mentais maternos e malformações congênitas do bebê, embora há muito estudada, ainda não é conclusiva. No entanto, o risco de ter anomalias congênitas parece ser maior em bebês de mães com transtornos psiquiátricos, principalmente esquizofrenia, em comparação com bebês de mães sem histórico de transtornos mentais 1,2,3,4,5,6. Estudos sobre os desfechos nas gestações de mulheres com transtornos psicóticos têm mostrado um aumento não só na incidência de malformações como também de complicações obstétricas em geral e mortalidade perinatal entre seus recém-nascidos 2,3,4,6,7,8,9. Os mecanismos causais dessa relação ainda são desconhecidos, mas suspeita-se que esse risco possa estar associado à administração de medicamentos antipsicóticos durante a gravidez 10,11, ao abuso de substâncias (álcool, tabaco e outras drogas) 12 , aos fatores relacionados ao estilo de vida (dieta e cuidado pré-natal insatisfatórios) 5 e às precárias condições socioeconômicas nas quais vivem essas mulheres 12 .

Ambos os temas desse estudo, malformações congênitas e transtornos mentais maternos, possuem relevância clínica e epidemiológica. Por malformação, anomalia ou anormalidade congênita entende-se a alteração morfológica ou estrutural, isolada ou múltipla, presente ao nascimento, sendo internacionalmente classificada segundo critérios anatômicos, funcionais 
ou genéticos e categorizada ainda como maiores e menores, segundo o grau de importância médica, cirúrgica ou cosmética, e impacto sobre a morbiletalidade do recém-nato ${ }^{13}$. A prevalência de malformações congênitas na população está em torno de $4 \% 14,15$, sendo mais frequente nas gestantes mais jovens e naquelas acima de 35 anos, nas tabagistas ou que fazem uso abusivo de álcool e outras drogas, e nas gestantes com poucas consultas pré-natais e desprovidas socioeconomicamente $5,12,14,15,16$.

O impacto dos defeitos congênitos, definição mais ampla de malformações congênitas que inclui anomalias funcionais não aparentes no recém-nascido e manifestadas tardiamente 17, vem aumentando progressivamente no Brasil, tendo passado da quinta para a segunda causa dos óbitos em menores de um ano entre 1980 e 2000 18,19,20. Em parte, esse aumento deve-se à diminuição do número de óbitos nesse grupo por causas infecciosas, resultando, assim, no aumento proporcional de mortes atribuíveis às malformações 19. Além da mortalidade, as anomalias congênitas também estão relacionadas à maior morbidade, risco de complicações clínicas, número de internações e gravidade das intercorrências. Estudos sobre morbidade infantil indicam que os transtornos genéticos e as malformações congênitas são responsáveis por até 25\% das hospitalizações em algumas cidades da América Latina 21.

Os transtornos mentais, por sua vez, afetam atualmente cerca de $10 \%$ da população mundial, com prevalência durante a vida de mais de $25 \%$, e estão entre as principais causas de anos de vida vividos com incapacidade (Years Lived with Disability - YLDs), sendo a depressão líder neste indicador 22,23. Embora os transtornos mentais acometam tanto os homens quanto as mulheres, alguns tipos como a depressão, por exemplo, são mais frequentes em mulheres. O período gravídico-puerperal é o período de maior prevalência desses transtornos na vida da mulher e a depressão é o transtorno mais frequente nesse período 24,25,26, afetando cerca de uma em cada cinco mães 27,28,29,30,31.

A importância da saúde mental materna aos poucos vem sendo clinicamente reconhecida, visto que os impactos dos transtornos mentais no período de gravidez e pós-parto não se restringem à saúde e bem-estar maternos, mas afetam também a saúde e o desenvolvimento do bebê. Sendo assim, o objetivo do presente artigo é realizar uma revisão sistemática com metanálise dos resultados encontrados pelos principais estudos epidemiológicos da última década que investigaram a associação entre transtornos mentais maternos e malformação congênita do bebê.

\section{Métodos}

Foi realizada uma revisão sistemática a respeito dos principais estudos epidemiológicos sobre a associação entre transtornos mentais maternos e malformações congênitas do bebê, segundo a metodologia descrita no PRISMA Statement 32. Para tanto, foram consultadas as seguintes bases de dados bibliográficos: PubMed/MEDLINE, ISIWEB, Scopus, LILACS e SciELO. A busca não foi restrita por idioma, mas os critérios de inclusão dos resumos para busca do texto completo foram: artigos publicados nos últimos dez anos (de 2000 a 2010) em inglês, espanhol e português; com desenho de estudo epidemiológico (seccional, caso-controle e coorte) e que estimaram medidas de associação entre os fatores estudados (exposição - transtornos mentais maternos e desfecho - malformações do bebê). A escolha da última década como período para a busca dos artigos deve-se ao objetivo deste estudo de avaliar as pesquisas mais recentes sobre o tema e, além disso, o que se tem observado é que somente nos últimos dez anos a literatura debruçou-se sobre a investigação científica da hipótese em questão, antes restrita a estudos de casos clínicos e sem amostras representativas.

$\mathrm{Na}$ estratégia de busca nas bases LILACS e SciELO foram utilizados os seguintes descritores, de acordo com sua definição no DeCS (Descritores em Ciências da Saúde): "mental disorders" OR "mental health" OR "psychotic disorders" OR "Schizophrenia” AND “congenital abnormalities". Nas bases PubMed/MEDLINE, ISIWEB e Scopus foram utilizadas palavras-chave definidas conforme sua descrição no MeSH (Medical Subject Headings), buscando-se: "mental disorder" OR "mental health" OR "psychotic disorders" OR "Schizophrenia" AND “congenital abnormalities" OR "birth defects". Diferentes palavras-chave foram utilizadas em cada base de dados devido às definições que cada uma das bases propõe para os descritores. Com esse procedimento, é possível que um maior número de artigos relacionados ao tema de interesse tenha sido capturado em cada base. Também foram revisadas as referências bibliográficas dos principais artigos encontrados e livros especializados. Dois revisores avaliaram os estudos de forma independente.

Estes artigos foram avaliados e pontuados conforme os critérios metodológicos propostos por Downs \& Black 33, aplicáveis ao delineamento dos artigos para avaliação de sua qualidade. Tais critérios avaliam a qualidade da informação, a validade interna (viéses e confundimentos), a validade externa e a capacidade de detecção de efeito significativo do estudo. O presente artigo utilizou a versão composta por 27 itens, sendo 
excluídos os itens relacionados a estudos experimentais. Deste modo, ao final, foram avaliados 17 itens, pontuando, no máximo, 18 pontos. Tais critérios foram utilizados por autores em artigos de revisão nacional 34,35 .

Os artigos foram então analisados quanto à qualidade metodológica, considerando-se os seguintes itens: hipóteses ou objetivos claramente descritos; desfecho claramente descrito na introdução ou metodologia; características dos participantes incluídos; distribuição das principais variáveis de confusão; principais resultados claramente descritos; informação sobre estimativas da variabilidade aleatória dos dados; características das perdas; informações sobre valores de probabilidade do desfecho; representatividade dos indivíduos incluídos no estudo; informação clara sobre resultados que não tenham sido baseados em hipóteses estabelecidas a priori; informação sobre ajuste na análise para diferentes durações de acompanhamento em estudos de coorte; igualdade do tempo entre a intervenção e o desfecho para casos e controles em estudos de caso-controle; adequação dos testes estatísticos; acurácia das medidas utilizadas para os principais desfechos; recrutamento dos participantes em diferentes grupos na mesma população e no mesmo período de tempo; inclusão adequada de variáveis de confusão na análise; e consideração das perdas de participantes durante o acompanhamento.

Foram incluídos no estudo somente os artigos (texto completo) que obtiveram pelo menos $50 \%$ da pontuação máxima da escala Downs \& Black (9 pontos). Foram extraídas as seguintes informações dos artigos selecionados: ano de publicação, local do estudo, tipo de dados (primários ou secundários), desenho de estudo, tamanho da amostra, instrumentos utilizados na avaliação dos transtornos mentais, prevalência de transtornos mentais, medidas de associação com malformações congênitas, e escore de avaliação metodológica.

A partir das estatísticas de associação referidas nos artigos, razão de chances (OR) e risco relativo (RR), bem como as respectivas medidas de variação (intervalos de confiança - IC), foram calculadas as medidas combinadas (RR e IC) através do modelo de efeitos fixos. Embora alguns artigos tenham fornecido o OR, esta medida foi considerada como um bom estimador do RR, já que ambos, desfecho e exposição, se tratavam de eventos raros. A heterogeneidade entre os resultados dos estudos foi avaliada através da estatística I2 que expressa a proporção da variação total que se deve à variação entre os estudos 36. Foi realizada análise de influência, excluindose da metanálise o único artigo com desenho de estudo diferente dos demais. O viés de publicação foi avaliado pelo gráfico de funil ${ }^{37}$. O pacote estatístico Stata versão 9.0 (Stata Corp., College Station, Estados Unidos) foi utilizado na análise de dados.

\section{Resultados}

A busca bibliográfica, segundo a estratégia estabelecida, resultou em 108 artigos (Figura 1). No entanto, apenas seis foram selecionados, segundo os critérios pré-estabelecidos (Tabela 1). Os demais foram excluídos por serem revisões de literatura, pesquisas qualitativas, estudos repetidos nas diferentes bases de dados ou por não serem referentes ao tema (muitos estudos encontrados pela busca eram pesquisas sobre as malformações e outras complicações obstétricas como fatores de risco para o desenvolvimento da esquizofrenia na vida adulta). Seis artigos foram excluídos por avaliarem transtornos mentais maternos como desfecho do diagnóstico de malformação congênita do bebê e não como fator de risco para tal.

Os seis estudos analisados eram provenientes dos seguintes países: Suécia, Dinamarca, Austrália e Israel. Quanto ao idioma, todos os artigos foram escritos em língua inglesa. Com relação ao desenho de estudo, a maioria dos estudos apresentados eram pesquisas baseadas em dados secundários, de base populacional, realizadas através do relacionamento entre bases de dados obstétricas e psiquiátricas, com exceção de um estudo longitudinal com dados primários.

No que se refere à avaliação metodológica dos estudos selecionados, destaca-se que todas as pesquisas analisadas obtiveram a pontuação máxima da escala Downs \& Black 33. Considerando que algumas questões eram específicas para determinados desenhos de estudos, o valor máximo da escala variou com a exclusão de um ou dois itens quando o estudo em análise não se enquadrava em tais critérios, sendo 18 pontos o escore máximo.

Com relação aos desenhos de estudo, é possível observar que em todos eles as amostras eram compostas tanto por crianças de mães com transtornos mentais como por um grupo de comparação de crianças de mães sem histórico de transtornos mentais. Nesses estudos, a avaliação dos transtornos era baseada nos registros médicos e de internações psiquiátricas, sendo que em apenas um estudo tais registros foram validados em uma amostra de 180 mulheres através de uma entrevista diagnóstica estruturada 2.

O principal transtorno mental avaliado em tais pesquisas era a esquizofrenia, embora alguns 
Fluxograma do resultado da busca nas fontes de informação, da seleção e inclusão dos artigos originais na revisão sistemática.

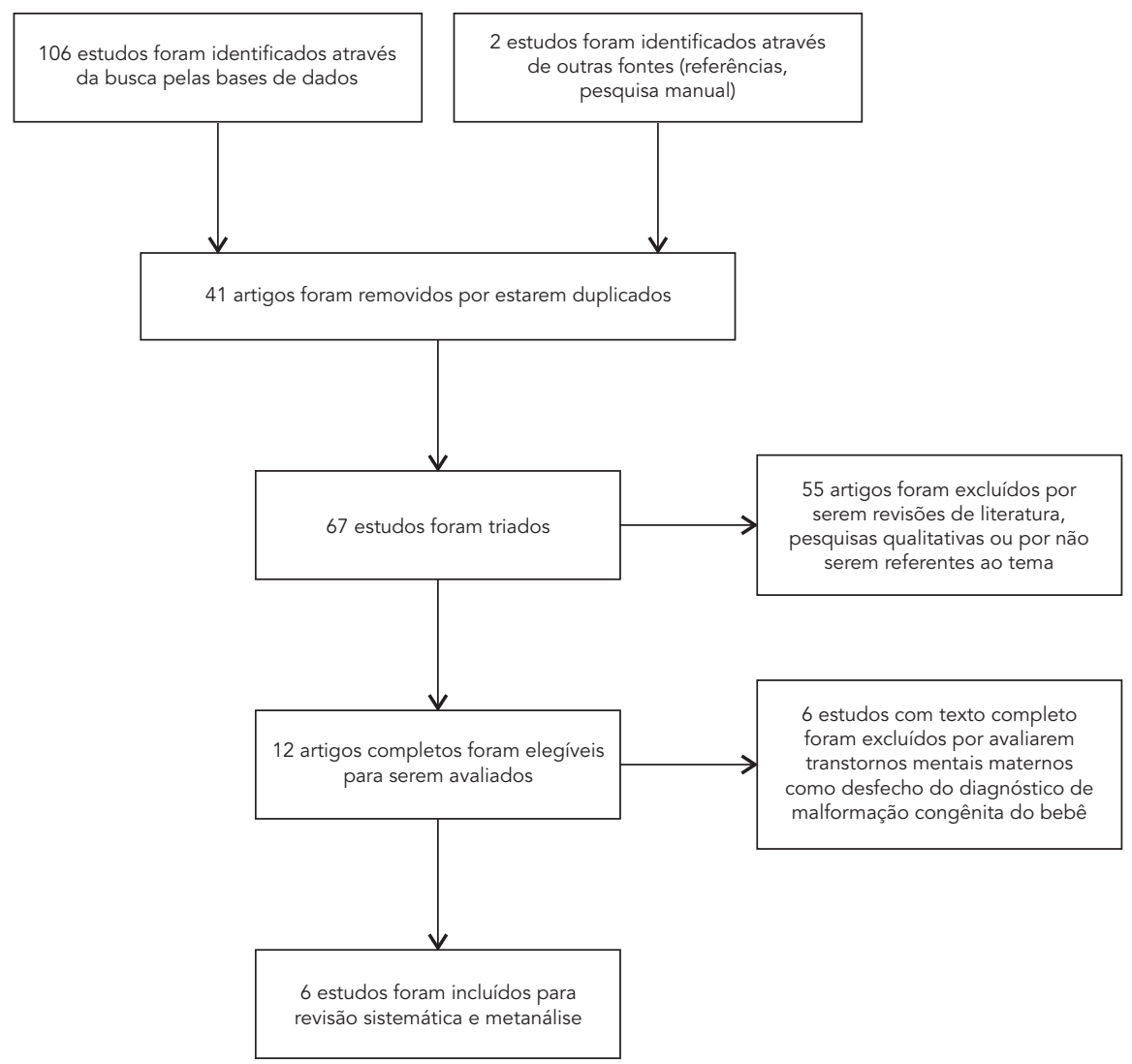

estudos incluíssem também transtornos afetivos 2,5. Algumas pesquisas estimaram a prevalência de esquizofrenia materna em coortes de nascimentos de base populacional, encontrando frequências abaixo de $1 \% 1,5$. No que diz respeito à relação entre transtornos mentais maternos e malformação congênita da criança, os estudos utilizaram diferentes medidas de associação, OR ou RR. No entanto, todos encontraram significância estatística, principalmente para esquizofrenia materna e risco de malformação, e em menor efeito para os transtornos afetivos.

Todos os artigos selecionados na revisão sistemática foram incluídos na metanálise, envolvendo um total de 4.801 crianças de mães com transtornos mentais e 430.420 crianças de mães sem histórico de transtornos psiquiátricos. A Figura 2 mostra que os resultados foram homogêneos, com todos os estudos revelando associação estatisticamente signiticativa entre transtornos mentais maternos e malformações congênitas do bebê. A medida combinada também revelou associação estatisticamente significativa entre exposição a transtornos mentais maternos e risco de malformações ( $R R=1,63$, IC: 1,27-1,99). Detaca-se que, apesar da homogeneidade dos estudos ( $\mathrm{I}^{2}=0,0 \%$; $\left.\mathrm{p}=0,43\right)$, foi realizada análise de influência, excluindo-se da metanálise o único artigo com desenho de estudo diferente dos demais ${ }^{3}$. Entretanto, não foram observadas alterações significativas nas medidas combinadas, como pode ser observado na Figura 3, optandose por incluir o estudo na análise. O gráfico de funil não sugere viés de publicação (Figura 4), no entanto, vale ressaltar que a análise fica limitada pelo pouco número de estudos encontrados, apenas seis, e os testes de Egger e Begg não foram utilizados, já que possuem baixo poder estatístico para amostras relativamente pequenas (menos do que 20 estudos) 37. 
Estudos sobre transtornos mentais maternos como um fator de risco para as anomalias congênitas.

\begin{tabular}{|c|c|c|c|c|c|c|c|}
\hline $\begin{array}{l}\text { Estudo } \\
\text { (Ano) }\end{array}$ & Local & Tipo de dados & Desenho do estudo & Amostra & $\begin{array}{l}\text { Instrumentos } \\
\text { (avaliação dos } \\
\text { transtornos } \\
\text { mentais) }\end{array}$ & $\begin{array}{c}\text { Prevalência de } \\
\text { transtornos mentais } \\
\text { maternos }\end{array}$ & $\begin{array}{l}\text { Associação com } \\
\text { malformações }\end{array}$ \\
\hline $\begin{array}{l}\text { Hizkiyahu } \\
\text { et al. } 1 \\
(2010)\end{array}$ & Israel & Secundários & $\begin{array}{c}\text { Coorte de } \\
\text { nascimentos de base } \\
\text { populacional (linkage } \\
\text { de bases de dados) }\end{array}$ & $\begin{array}{c}97 \text { crianças de mães } \\
\text { com esquizofrenia } \\
\text { e transtornos } \\
\text { esquizoafetivos e } \\
186.457 \text { crianças } \\
\text { de mães sem } \\
\text { transtornos mentais }\end{array}$ & $\begin{array}{l}\text { Registros baseados } \\
\text { nas admissões } \\
\text { hospitalares }\end{array}$ & $\begin{array}{c}\text { Esquizofrenia } \\
\text { e transtornos } \\
\text { esquizoafetivos: } \\
0,05 \%\end{array}$ & $\begin{array}{c}\text { Esquizofrenia } \\
\text { e transtornos } \\
\text { esquizoafetivos } \\
\text { e malformações } \\
\text { congênitas: } \\
\text { p = 0,017; OR = 2,3; } \\
\text { IC95\%: } 1,3-4,4\end{array}$ \\
\hline $\begin{array}{l}\text { Webb et } \\
\text { al. } 5 \text { (2008) }\end{array}$ & Dinamarca & Secundários & $\begin{array}{c}\text { Coorte de } \\
\text { nascimentos de base } \\
\text { populacional (linkage } \\
\text { de bases de dados) }\end{array}$ & $\begin{array}{c}102 \text { crianças de } \\
\text { mães admitidas com } \\
\text { transtornos mentais } \\
\text { e } 3.765 \text { crianças de } \\
\text { mães sem admissão } \\
\text { por transtornos } \\
\text { mentais }\end{array}$ & $\begin{array}{c}\text { Registros baseados } \\
\text { na CID (admissões } \\
\text { hospitalares) }\end{array}$ & $\begin{array}{l}\text { Esquizofrenia: } 0,2 \% ; \\
\text { transtornos afetivos: } \\
0,6 \% \text {; transtornos } \\
\text { relacionados ao uso } \\
\text { de álcool/drogas: } \\
\text { 0,4\%; todos os } \\
\text { transtornos mentais: } \\
2,4 \%\end{array}$ & $\begin{array}{c}\text { Esquizofrenia e } \\
\text { defeitos congênitos } \\
\text { fatais: } \mathrm{RR}=2,34 \text {; } \\
\text { IC95\%: 1,45- } \\
\text { 3,77. Transtornos } \\
\text { afetivos e defeitos } \\
\text { congênitos fatais: } \\
\text { RR = 1,54; IC95\%: } \\
\text { 1,05-2,27 }\end{array}$ \\
\hline $\begin{array}{l}\text { Jablensky } \\
\text { et al. } 2 \\
(2005)\end{array}$ & Austrália & Secundários & $\begin{array}{c}\text { Coorte de } \\
\text { nascimentos de base } \\
\text { populacional (linkage } \\
\text { de bases de dados) }\end{array}$ & $\begin{array}{l}3.174 \text { crianças } \\
\text { de mães com } \\
\text { esquizofrenia } \\
\text { ou transtornos } \\
\text { afetivos e } 3.129 \\
\text { crianças de mães } \\
\text { sem transtornos } \\
\text { psiquiátricos }\end{array}$ & $\begin{array}{c}\text { Registros } \\
\text { psiquiátricos } \\
\text { (diagnósticos de } \\
\text { esquizofrenia e } \\
\text { transtornos afetivos } \\
\text { foram validados em } \\
\text { uma amostra de } 180 \\
\text { mulheres através } \\
\text { de uma entrevista } \\
\text { diagnóstica semi- } \\
\text { estruturada - } \\
\text { OPCRIT) }\end{array}$ & $\mathrm{NI}$ & $\begin{array}{c}\text { Esquizofrenia e } \\
\text { malformações } \\
\text { cardiovasculares: } \\
\text { OR = 2,55; IC95\%: } \\
1,19-5,46\end{array}$ \\
\hline $\begin{array}{l}\text { Schneid- } \\
\text { Kofman et } \\
\text { al. } 6 \text { (2008) }\end{array}$ & Israel & Secundários & $\begin{array}{c}\text { Coorte de } \\
\text { nascimentos de base } \\
\text { populacional (linkage } \\
\text { de bases de dados) }\end{array}$ & $\begin{array}{c}181.479 \text { partos: } 607 \\
\text { partos de mulheres } \\
\text { com histórico } \\
\text { de transtornos } \\
\text { psiquiátricos e } \\
180.872 \text { partos } \\
\text { de mulheres } \\
\text { sem transtornos } \\
\text { psiquiátricos }\end{array}$ & $\begin{array}{l}\text { Registros } \\
\text { psiquiátricos } \\
\text { (admissões } \\
\text { hospitalares) }\end{array}$ & $\mathrm{NI}$ & $\begin{array}{c}\text { Transtornos mentais } \\
\text { na gravidez e } \\
\text { malformações } \\
\text { congênitas: } \\
\text { OR =1,4; IC95\%: } \\
1,01-1,9 ; p=0,03\end{array}$ \\
\hline $\begin{array}{l}\text { Schubert } \\
\& \text { McNeil } 3 \\
\text { (2004) }\end{array}$ & Suécia & Primários & Longitudinal & $\begin{array}{c}75 \text { crianças de mães } \\
\text { com transtornos } \\
\text { psicóticos e } 91 \\
\text { crianças de mães } \\
\text { sem histórico } \\
\text { de transtornos } \\
\text { psicóticos }\end{array}$ & $\begin{array}{l}\text { Registros médicos } \\
\text { de clínicas pré-natais } \\
\text { no sudoeste da } \\
\text { Suécia }\end{array}$ & $\mathrm{NI}$ & $\begin{array}{c}\text { Esquizofrenia e } \\
\text { anormalidades } \\
\text { neurológicas: } \\
\text { OR = 4,74; IC95\%: } \\
1,61-13,89 ; \\
\text { P }=0,005\end{array}$ \\
\hline
\end{tabular}

(continua) 
Tabela 1 (continuação)

\begin{tabular}{|c|c|c|c|c|c|c|c|}
\hline $\begin{array}{l}\text { Estudo } \\
\text { (Ano) }\end{array}$ & Local & Tipo de dados & Desenho do estudo & Amostra & $\begin{array}{l}\text { Instrumentos } \\
\text { (avaliação dos } \\
\text { transtornos } \\
\text { mentais) }\end{array}$ & $\begin{array}{c}\text { Prevalência de } \\
\text { transtornos mentais } \\
\text { maternos }\end{array}$ & $\begin{array}{l}\text { Associação com } \\
\text { malformações }\end{array}$ \\
\hline $\begin{array}{l}\text { Bennedsen } \\
\text { et al. } 4 \\
\text { (2001) }\end{array}$ & Dinamarca & Secundários & $\begin{array}{l}\text { Coorte de } \\
\text { nascimentos de base } \\
\text { populacional (linkage } \\
\text { de bases de dados) }\end{array}$ & $\begin{array}{l}746 \text { crianças de mães } \\
\text { com esquizofrenia e } \\
56.106 \text { crianças na } \\
\text { população em geral }\end{array}$ & $\begin{array}{c}\text { Registros } \\
\text { psiquiátricos } \\
\text { nacionais (mães } \\
\text { com pelo menos } \\
\text { uma internação } \\
\text { psiquiátrica } \\
\text { por transtornos } \\
\text { psicóticos) }\end{array}$ & $\mathrm{NI}$ & $\begin{array}{c}\text { Esquizofrenia e } \\
\text { malformações } \\
\text { congênitas: } \\
\text { RR = 1,70; IC95\% } \\
1,04-2,77\end{array}$ \\
\hline
\end{tabular}

CID: Classificação Internacional de Doenças; NI: não informado; OPCRIT: Inventário de Critérios Operacionais para Doenças Psicóticas.

Figura 2

Risco relativo (RR) de malformações congênitas em crianças de mães com transtornos mentais em comparação com crianças de mães sem transtornos mentais, com intervalo de $95 \%$ de confiança (IC95\%).

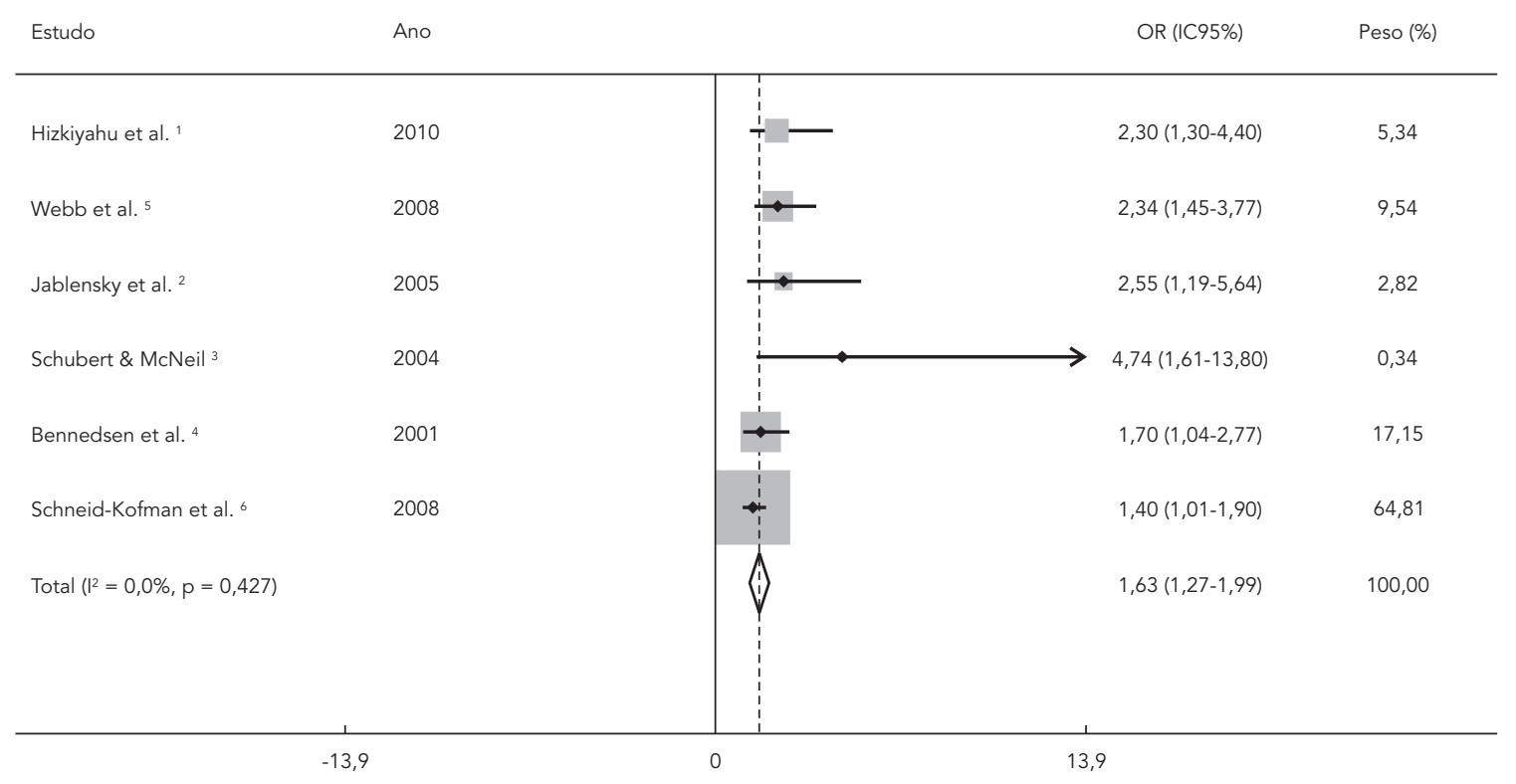

\section{Discussão}

Os resultados do presente estudo confirmam a associação entre exposição a transtornos mentais maternos e risco de malformações congênitas no bebê: crianças de mães com transtornos mentais maiores tiveram risco $63 \%$ maior de nascer com malformações em comparação com crianças de mães sem transtornos mentais
$(\mathrm{RR}=1,63$, IC: 1,27-1,99). Sendo assim, estes achados corroboraram a hipótese de uma associação entre saúde mental materna durante o período gravídico e suas repercussões na saúde do bebê, o que torna o tema de fundamental consideração para o campo da saúde materno-infantil.

Além da significativa homogeneidade dos estudos observada na análise, pode-se dizer que os resultados encontrados por estas pesquisas 
Figura 3

Forest plot das medidas isoladas e combinadas e respectivos intervalos de $95 \%$ de confiança, excluindo-se o estudo de Schubert \& McNeil 3 .

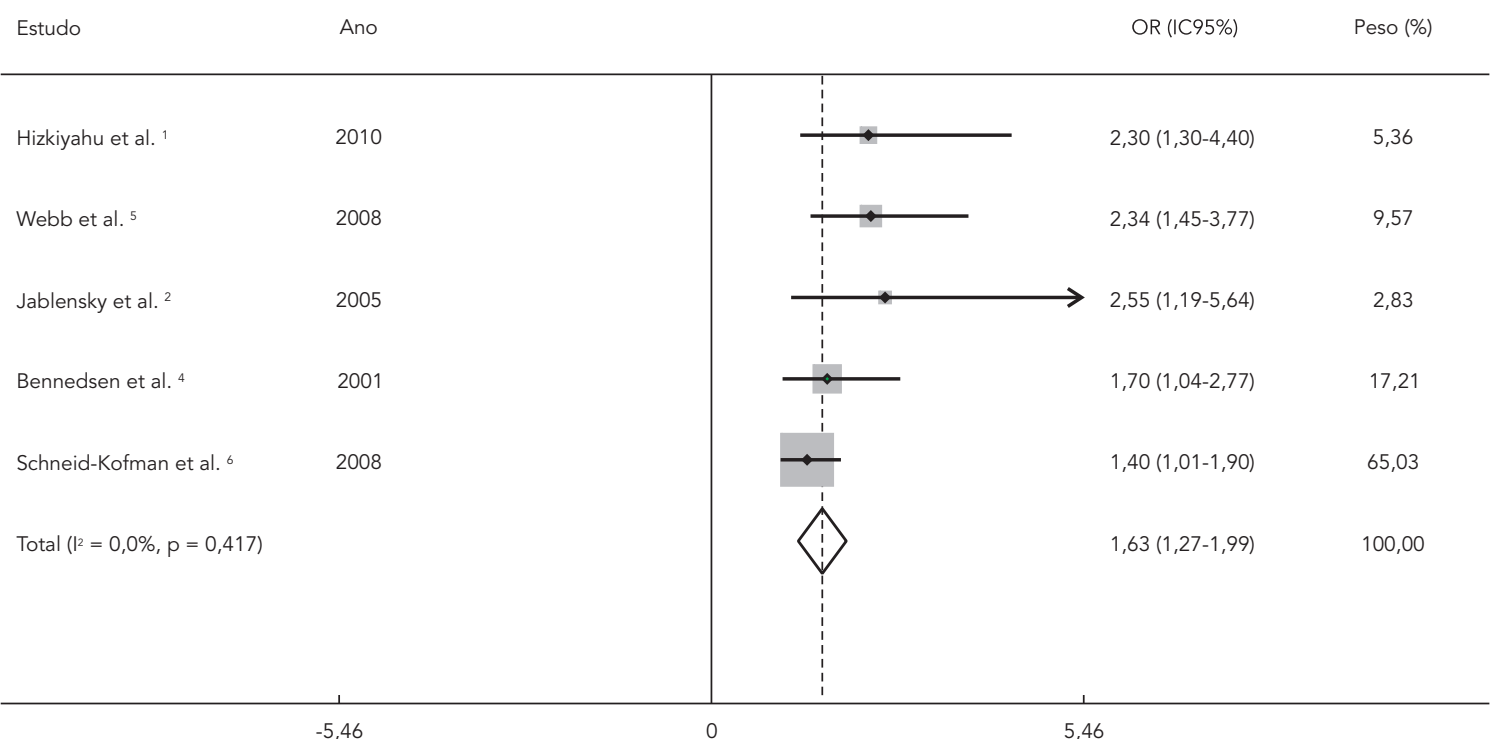

Figura 4

Gráfico de funil dos estudos sobre transtornos mentais maternos e risco de malformações congênitas do bebê.

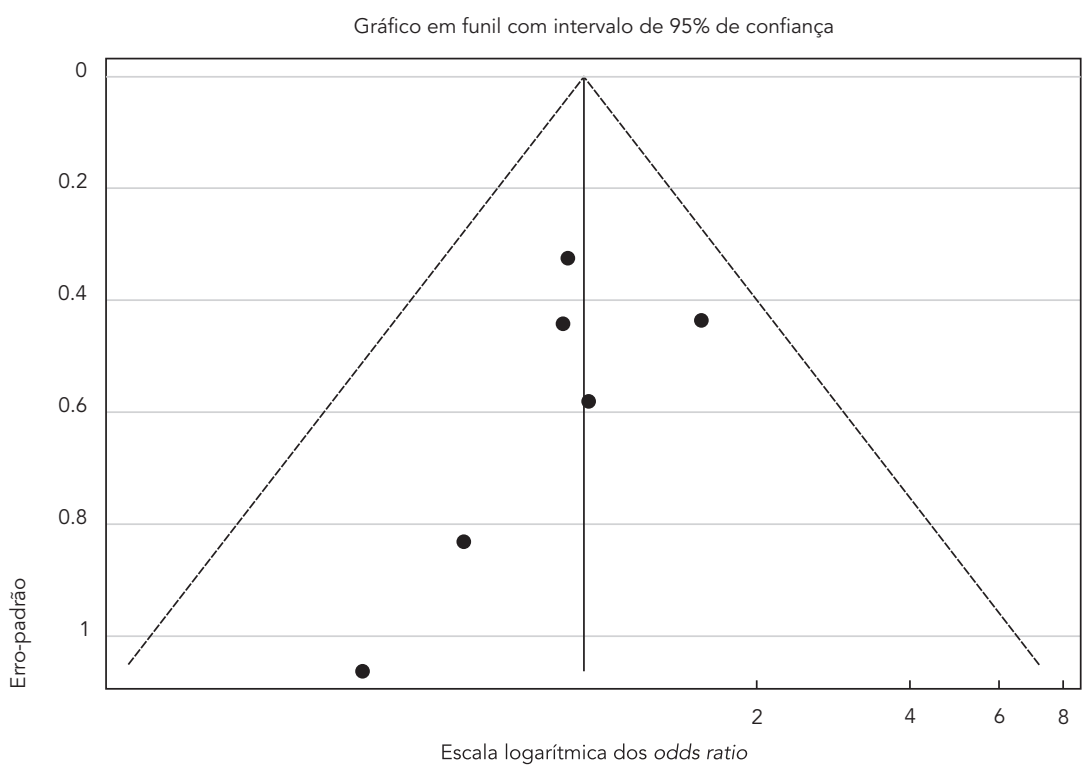


podem ser considerados de boa fidedignidade, já que apresentaram metodologias semelhantes e bem delimitadas, com amostras de base populacional e avaliação tanto da exposição (transtornos mentais) quanto do desfecho (malformações congênitas), baseada no diagnóstico médico presente nos registros hospitalares, obtendo inclusive o escore máximo na avaliação metodológica segundo os critérios de Downs \& Black 33. Apenas um desses estudos utilizou uma metodologia diferente, baseada em dados primários, com desenho longitudinal e amostras menores, no entanto esse estudo também obteve escore máximo na referida escala de avaliação metodológica e sua exclusão da análise não modificou a medida combinada, já que teve um peso pequeno devido ao reduzido tamanho amostral ${ }^{3}$. Embora, o gráfico de funil não apresente indícios de viés de publicação, é preciso destacar que foram poucos artigos encontrados e todos encontraram algum grau de associação entre os fatores estudados, o que pode comprometer a análise.

Com relação aos transtornos mentais avaliados, observou-se que mesmo naqueles estudos que analisaram vários transtornos mentais maiores, a esquizofrenia se destacou, apresentando significativa associação com malformações congênitas, sendo o risco ou a chance encontrada de ter algum tipo de malformação pelo menos duas vezes maior em crianças de mães esquizofrênicas 2,3,4,5. Apenas dois estudos analisaram conjuntamente esquizofrenia e transtornos afetivos encontrando da mesma forma associação significativa com o desfecho em questão 1,6.

De uma maneira geral, não houve restrição quanto aos tipos de malformações investigadas nos estudos, apenas uma pesquisa avaliou somente malformações do sistema nervoso 3 . Aqueles estudos que estratificaram a análise por tipos de malformações encontraram maiores frequências de malfomações cardiovasculares e de defeitos congênitos fatais entre as crianças de mães esquizofrênicas, comparadas às crianças de mães sem o transtorno 2,5 . No entanto, a alta frequência encontrada de malformações cardiovasculares pode se dever ao fato de estas serem umas das mais comuns entre as malformações. Ainda não há hipóteses plausíveis para a associação da esquizofrenia materna com um ou outro determinado tipo de malformação.

As pesquisas analisadas encontraram associação também da esquizofrenia e transtornos afetivos maternos com outras complicações obstétricas, além das malformações fetais. Hemorragias, anormalidades placentárias e sofrimento fetal foram significativamente mais frequentes em gestantes esquizofrênicas e com transtornos afetivos 2 , enquanto que a mortalidade perina- tal e o baixo peso estiveram associados apenas à esquizofrenia materna 1,2,4,6. Entretanto, na literatura já existem vários estudos que revelaram a associação da prematuridade e do baixo peso ao nascer com outros transtornos mentais durante a gravidez, principalmente com a depressão e transtornos de ansiedade 38,39,40.

Os possíveis mecanismos causais da associação entre transtorno mental materno e complicações obstétricas e malformações congênitas destacados pelos estudos analisados incluem os efeitos teratogênicos do uso de antipsicóticos e de substâncias como álcool, tabaco e outras drogas durante a gravidez 10,11,12. Além disso, fatores relacionados ao estilo de vida dessas mulheres com transtornos psiquiátricos, como dieta precária, vida sedentária e maus hábitos de saúde em geral também podem estar envolvidos 5 .

A mulher com transtornos mentais no período gestacional, devido aos próprios sintomas psiquiátricos, pode apresentar muitas vezes uma menor preocupação com seu estado de saúde, tendo dificuldades para seguir as orientações médicas e realizar adequadamente o acompanhamento pré-natal, o que por si só já está associado a inúmeros riscos obstétricos e neonatais 41 . Outra questão importante ressaltada é o fato de que essas pacientes psiquiátricas vivem na maioria das vezes em precárias condições socioeconômicas, contando com poucos recursos materiais e sociais, o que pode ter repercussões diretas no bom andamento da gravidez e no desenvolvimento do bebê 12 .

Pelo fato de esses estudos se basearem em dados secundários, o controle das variáveis de confusão pode ser prejudicado, já que muitas vezes não estão disponíveis informações sobre o status socioeconômico das mulheres, o uso de álcool, tabaco e outras drogas durante a gravidez, bem como informações sobre o tratamento psicofarmacológico. Além disso, um estudo revelou que as mães esquizofrênicas tinham em média mais idade que as mães sem o transtorno, e ainda que doenças como diabetes e hipertensão eram mais frequentes em mulheres com transtornos mentais severos, como a esquizofrenia, em comparação com mulheres sem transtornos mentais 1 . Tais doenças, bem como a idade materna avançada, são fatores de risco já reconhecidos para complicações obstétricas e malformações congênitas do bebê.

Um outro achado desses estudos diz respeito à prevalência de esquizofrenia, encontrada abaixo de $0,5 \%$, estando próxima à frequência conhecida para este transtorno na população geral, que varia de 0,5 a $1,5 \% 42$, sendo dessa forma substancial se considerarmos que se trata de uma população específica de mães. Embo- 
ra esta prevalência possa parecer pequena, não se pode esquecer que os períodos de gravidez e pós-parto além de constituírem uma fase de risco para transtornos mentais na vida da mulher, compreendem ainda um momento crucial para a formação e o desenvolvimento da criança. Um dos estudos avaliados acompanhou os descendentes de mães com transtornos psicóticos e de mães sem história de transtornos psiquiátricos do nascimento até a vida adulta, encontrando inclusive uma associação significativa entre a esquizofrenia materna e distúrbios do neurodesenvolvimento na criança ${ }^{3}$.

Dessa forma, transtornos mentais maternos não afetam só a saúde e o bem-estar da mãe, podendo interferir diretamente no autocuidado materno, tendo com isso repercussões negativas na saúde e desenvolvimento do feto durante a gravidez. Além disso, também podem comprometer o vínculo afetivo da mãe com seu bebê $43 \mathrm{e}$ a capacidade materna de cuidado, aumentando inclusive o risco de infecções e desnutrição infantil 44 , de comprometimento do crescimento da criança, expresso no baixo peso e altura para a idade 40,45 , e até mesmo problemas comportamentais e transtornos mentais na vida adulta 46 .

Essa questão torna-se ainda mais delicada quando se trata de um bebê com malformação congênita, que inevitavelmente necessitará de maior atenção e assistência materna. O cuidado materno adequado nesse caso pode ser de fundamental importância para a sobrevivência e desenvolvimento da criança. Embora alguns estudos revelem que as mães tendem a manter o apego em relação ao bebê mesmo diante do diagnóstico de malformações 47,48 , a presença de transtornos mentais maternos pode interferir diretamente na vinculação afetiva da mãe com o bebê, o que poderá dificultar o cuidado materno, agravando uma situação que já é crítica.

Apesar da relevância dessa questão e de todo esforço empreendido na busca por artigos sobre o tema nas diferentes bases de dados, foram encontrados poucos artigos. A avaliação da qualidade metodológica dos artigos, com base nos critérios estabelecidos por Downs \& Black 33 , permitiu orientar os revisores quanto às limita- ções de cada artigo avaliado, possibilitando uma leitura mais crítica dos estudos publicados. Com isso, é possível destacar as principais limitações dos estudos analisados, permitindo apontar novas perspectivas para futuras pesquisas sobre $o$ tema, principalmente em termos nacionais.

São necessárias mais pesquisas que testem a associação entre os fatores em questão, com desenhos de estudo diferenciados, que incluam dados primários, permitindo o controle de possíveis variáveis de confusão, e que avaliem também transtornos mentais menores e outros possíveis desfechos na gravidez, assegurando a confirmação do diagnóstico, tanto da exposição quanto do desfecho, através de instrumentos padronizados e validados, já que não se pode afastar as possibilidades de erros nos registros das bases de dados. Como proposta para novos estudos enfatiza-se a necessidade de pesquisas que investiguem associações entre esquizofrenia materna e malformações específicas e também que identifiquem o mecanismo pelo qual tal associação opera. Cabe destacar ainda a ausência de estudos brasileiros sobre o tema.

Assim, o presente artigo traz à tona a discussão de uma importante questão no que diz respeito à saúde materno-infantil: a saúde mental materna. Como já comprovado em estudos anteriores, os transtornos mentais afetam pelo menos $10 \%$ das mães 24 , sejam eles anteriores ou decorrentes do período de gravidez e puerpério, repercutindo não apenas na saúde materna como também no desenvolvimento do bebê. Tendo em vista a escassez de estudos sobre o tema, novas pesquisas são necessárias para aprimorar o conhecimento da prevalência desses transtornos antes e durante a gestação e de sua associação com o risco de malformação e de outras complicações obstétricas, visando fundamentar políticas públicas de saúde materno-infantil, que contemplem novas práticas assistenciais, como a implementação da assistência em saúde mental na atenção pré-natal, o que permitiria a detecção precoce, adequado tratamento e manejo dessas pacientes e mesmo a prevenção dos transtornos mentais maternos posteriores, minimizando com isso os riscos à saúde da criança. 


\section{Resumo}

O risco de ter malformações parece ser maior em bebês de mães com transtornos mentais em comparação com bebês de mães sem histórico de transtornos psiquiátricos. O objetivo deste artigo foi realizar uma metanálise dos estudos sobre a associação entre transtornos mentais maternos e malformações congênitas. A revisão consistiu na busca de artigos nas bases MEDLINE, ISIWEB, Scopus, LILACS e SciELO, utilizando-se os descritores: "mental disorders" OR "mental health" OR "psychotic disorders" OR "schizophrenia" AND "congenital abnormalities" $O R$ "birth defects". Foram localizados 108 estudos, sendo selecionados cinco artigos de acordo com os critérios estabelecidos. Estes artigos foram incluídos na metanálise, envolvendo um total de 4.194 crianças de mães com transtornos mentais e 249.548 crianças de mães sem tais transtornos. A medida combinada revelou associação significativa entre exposição a transtornos mentais maternos e risco de malformações ( $R R=2,06$, IC95\%: 1,46-2,67). O presente estudo evidencia a relação entre saúde mental materna durante a gravidez e suas repercussões na saúde do bebê.

Anormalidades Congênitas; Transtornos Mentais; Esquizofrenia; Metanálise

\section{Colaboradores}

P. K. Pereira contribuiu com a concepção e projeto, análise e interpretação dos dados, redação do artigo, e aprovação final da versão a ser publicada. L. A. Lima, M. M. F. Magnanini e L. F. Legay contribuiu com a análise e interpretação dos dados, revisão crítica do conteúdo e aprovação da versão final do manuscrito. G. M. Lovisi contribuiu com a concepção e projeto, análise e interpretação dos dados, revisão crítica do conteúdo e aprovação da versão final do manuscrito.

\section{Referências}

1. Hizkiyahu R, Levy A, Sheiner E. Pregnancy outcome of patients with schizophrenia. Am J Perinatol 2010; 27:19-24.

2. Jablensky AV, Morgan V, Zubrick SR, Bower C, Yellachich L. Pregnancy, delivery, and neonatal complications in a population cohort of women with schizophrenia and major affective disorders. Am J Psychiatr 2005; 162:79-91.

3. Schubert EW, McNeil TF. Prospective study of neurological abnormalities in offspring of women with psychosis: birth to adulthood. Am J Psychiatr 2004; 161:1030-7.

4. Bennedsen B, Mortensen PB, Olesen AV, Henriksen TB. Congenital malformations, stillbirths, and infant deaths among children of women with schizophrenia. Arch Gen Psychiatr 2001; 58:674-9.
5. Webb RT, Pickles AR, King-Hele SA, Appleby L, Mortensen PB, Abel KM. Parental mental illness and fatal birth defects in a national birth cohort. Psychol Med 2008; 38:1495-503.

6. Schneid-Kofman N, Sheiner E, Levy A. Psychiatric illness and adverse pregnancy outcome. Int J Gynaecol Obstet 2008; 101:53-6.

7. Sobel DE. Infant mortality and malformations in children of schizophrenic women. Psychiatr Q 1961; 35:60-5.

8. Rieder RO, Rosenthal D, Wender P, Blumenthal H. The offspring of schizophrenics: fetal and neonatal deaths. Arch Gen Psychiatr 1975; 32:200-11.

9. Modrzewska K. The offspring of schizophrenic parents in a North Swedish isolate. Clin Genet 1980; 17:191-201. 
10. Friedman JM, Polifka JE. The effects of neurologic and psychiatric drugs on the fetus and nursing infant. Baltimore: Johns Hopkins University Press; 1998.

11. Altshuler LL, Cohen L, Szuba MP, Burt VK, Gitlin M, Mintz J. Pharmacologic management of psychiatric illness during pregnancy: dilemmas and guidelines. Am J Psychiatr 1996; 153:592-606.

12. Bennedsen B. Adverse pregnancy outcome in schizophrenic women: occurrence and risk factors. Schizophr Res 1998; 33:1-26.

13. Moore KL, Persaud TVN. Embriologia clínica. Defeitos congênitos humanos. Rio de Janeiro: Editora Guanabara Koogan; 2000.

14. Stoll C, Alembik Y, Dott B, Roth MP. Impact of prenatal diagnosis on livebirth prevalence of children with congenital anomalies. Ann Genet 2002; 45:115-21.

15. Penchaszadeh VB. Estabelecimiento de servicios integrales de genética en los países en desarrollo: América Latina. Bol Oficina Sanit Panam 1993; 115:39-47.

16. Basso O, Olsen J, Christensen K. Recurrence risk of congenital anomalies - the impact of paternal, social and environmental factors: a population study in Denmark. Am J Epidemiol 1999; 150:598-604.

17. Organização Pan-Americana da Saúde. Prevenção e controle de enfermidades genéticas e os defeitos congênitos: relatório de um grupo de consulta. Washington DC: Organização Pan-Americana da Saúde; 1984. (Publicação Científica, 460).

18. Secretaria de Estado de Saúde do Rio de Janeiro. Indicadores e informações em saúde. http://www. saude.rj.gov.br/informacoes/obitos.shtml (acessado em 20/Nov/2010).

19. Horovitz DDG, Llerena Jr. JC, Mattos RA. Atenção aos defeitos congênitos no Brasil: panorama atual. Cad Saúde Pública 2005; 21:1055-64.

20. Rosano A, Botto LD, Botting B, Mastroiacovo P. Infant mortality and congenital anomalies from 1950 to 1994: an international perspective. J Epidemiol Community Health 2000; 54:660-6.

21. Cunha AJB. Orientação genética-clínica em medicina fetal. In: Sanchez RC, Saito M, Isfer EV, organizadores. Medicina fetal diagnóstico pré-natal e conduta. Rio de Janeiro: Revinter; 1996. p. 1-19.

22. World Health Organization. The World Health Report - Mental health: new understanding, new hope. Geneva: World Health Organization; 2001.

23. World Health Organization. Integrating mental health into primary care - a global perspective. Geneva: World Health Organization; 2008.

24. Bennett HA, Einarson A, Tadd A, Koren G, Einarton TR. Prevalence of depression during pregnancy: systematic review. Obstet Gynaecol 2004; 103:698709 .

25. Camacho RS, Cantinelli FS, Ribeiro CS, Cantilino A, Gonsales BK, Braguittoni E, et al. Transtornos psiquiátricos na gestação e no puerpério: classificação, diagnóstico e tratamento. Rev Psiquiatr Clín (São Paulo) 2006; 33:92-102.

26. Andrade LHSG, Viana MC, Silveira CM. Epidemiology of women's psychiatric disorders. Rev Psiquiatr Clín (São Paulo) 2006; 33:43-54.
27. Alami KM, Kadri N, Berrada S. Prevalence and psychosocial correlates of depressed mood during pregnancy and after chilbirth in a Moroccan sample. Arch Womens Ment Health 2006; 9:343-6.

28. Faisal-Cury A, Menezes PR. Prevalence of anxiety and depression during pregnancy in a private setting sample. Arch Womens Ment Health 2007; 10:25-32.

29. Limlomwongse NN, Liabsuetrakul T. Cohort study of depressive moods in Thai women during late pregnancy and 6-8 weeks of postpartum using the Edinburgh Postnatal Depression Scale (EPDS). Arch Womens Ment Health 2006; 9:131-8.

30. Marcus SM, Flynn HA, Blow FC, Barry KL. Depressive symptoms among pregnant women screened in obstetrics settings. J Womens Health 2003; 12:373-80

31. Patel V, Rodrigues M, Desouza N. Gender, poverty and postnatal depression: a study of mothers in Goa, India. Am J Psychiatr 2002; 159:43-7.

32. Liberati A, Altman DG, Tetzlaff J, Mulrow C, Gøtzsche PC, Ioannidis JPA, et al. The PRISMA statement for reporting systematic reviews and metaanalyses of studies that evaluate health care interventions: explanation and elaboration. PLoS Med 2009; 6:1-28.

33. Downs SH, Black N. The feasibility of creating a checklist for the assessment of the methodological quality both of randomised and non-randomised studies of health care interventions. J Epidemiol Community Health 1998; 52:377-84.

34. Araujo DMR, Vilarim MM, Sabroza AR, Nardi AE. Depressão no período gestacional e baixo peso ao nascer: uma revisão sistemática da literatura. Cad Saúde Pública 2010; 26:219-27.

35. Rossi CE, Vasconcelos FAG. Peso ao nascer e obesidade em crianças e adolescentes: uma revisão sistemática. Rev Bras Epidemiol 2010; 13:246-58.

36. Higgins JPT, Thompson SG, Deeks JJ, Altman DG. Measuring inconsistency in meta-analyses. BMJ 2003; 327:557-60.

37. Egger M, Davey Smith G, Schneider M, Minder C. Bias in meta-analysis detected by a simple, graphical test. BMJ 1997; 315:629-34.

38. Kramer MS, Lydon J, Séguin L, Goulet L, Kahn SR, McNamara H, et al. Stress pathways to spontaneous preterm birth: the role of stressors, psychological distress and stress hormones. Am J Epidemiol 2009; 169:1319-26.

39. Patel V, Prince M. Maternal psychological morbidity and low birth in India. Br J Psychiatr 2006; 188:284-5.

40. Patel V, Rahman A, Jacob, KS, Hughes M. Effect of maternal mental health on infant growth in low income countries: new evidence from South Asia. BMJ 2004; 328:820-3.

41. Carvalho PI, Pereira PMH, Frias PG, Vidal AS, Figueiroa JN. Fatores de risco para a mortalidade neonatal em coorte hospitalar de nascidos vivos. Epidemiol Serv Saúde 2007; 16:185-94.

42. American Psychiatric Association. Manual diagnóstico e estatístico de transtornos mentais. Porto Alegre: Editora Artes Médicas; 1994. 
43. Schmidt EB, Argimon IIL. Vinculação da gestante e apego materno fetal. Paidéia (Ribeirão Preto) 2009; 19:211-20.

44. Carvalhaes MAB, Benício MHD’A. Capacidade materna de cuidar e desnutrição infantil. Rev Saúde Pública 2002; 36:188-97.

45. Rahman A, Harrington R, Bunn J. Can maternal depression increase infant risk of illness and growth impairment in developing countries? Child Care Health Dev 2002; 28:51-6.

46. Huot RL, Brennan PA, Stowe ZN, Plotsky PM, Walker EF. Negative affect in offspring of depressed mothers is predicted by infant cortisol levels at 6 months and maternal depression during pregnancy, but not postpartum. Ann NY Acad Sci 2004; 1032:234-6.
47. Vasconcelos L, Petean EBL. O impacto da malformação fetal: indicadores afetivos e estratégias de enfrentamento das gestantes. Psicol Saúde Doenças 2009; 10:69-82.

48. Gomes AG, Piccinini CA. Impressões e sentimentos das gestantes sobre a ultra-sonografia e suas implicações para a relação materno-fetal no contexto da anormalidade fetal. Psico (Porto Alegre) 2007; 38:67-76.

Recebido em 13/Dez/2010

Versão final reapresentada em 10/Ago/2011

Aprovado em 29/Ago/2011 